\title{
ESTUDO CINÉTICO DO CRAQUEAMENTO TÉRMICO E CATALÍTICO DO ÁCIDO MIRÍSTICO VIA ANÁLISE TERMOGRA VIMÉTRICA
}

\author{
E.D.V.BRUCE ${ }^{1}$, C.M.T.ALMEIDA ${ }^{2}$, J.G.P.A.FILHO ${ }^{3}$, R.T.F.FRETY ${ }^{4}$, C.M.B.M.BARBOSA ${ }^{5}$. \\ 1,2,3,4,5 Universidade Federal de Pernambuco, Departamento de Engenharia Química \\ E-mail para contato: eniobruce@ hotmail.com
}

RESUMO - O aproveitamento de óleos residuais com alto teor de ácidos graxos livres vem se tornando um tema importante para obtenção de biocombustíveis. Neste trabalho foi estudada a cinética de craqueamento térmico e catalítico do ácido mirístico como um composto modelo de ácido graxo. O craqueamento catalítico foi realizado usando catalisadores com $10 \%$ de molibdênio suportado em $\gamma-\mathrm{Al}_{2} \mathrm{O}_{3}$ e $\mathrm{SiO}_{2}-\mathrm{Al}_{2} \mathrm{O}_{3}$ comerciais. $\mathrm{O}$ craqueamento térmico foi realizado na faixa de temperatura de $30^{\circ} \mathrm{C}$ a $700^{\circ} \mathrm{C}$ com taxas de aquecimento de $3,7,10$ e $15^{\circ} \mathrm{C} / \mathrm{min}$. O craqueamento foi realizado com razão mássica catalisador:ácido igual a 5:1. O estudo cinético foi realizado por meio de análises termogravimétricas, utilizando o método de Flynn-Wall-Ozawa obtendo energias de ativação média de $98,9 \mathrm{~kJ} / \mathrm{mol}$ para o processo térmico, $64,9 \mathrm{~kJ} / \mathrm{mol}$ e $85,1 \mathrm{~kJ} / \mathrm{mol}$ no processo catalítico sob $10 \% \mathrm{Mo} / \gamma-\mathrm{Al}_{2} \mathrm{O}_{3}$ e $10 \% \mathrm{Mo} / \mathrm{SiO}_{2}-\mathrm{Al}_{2} \mathrm{O}_{3}$.

\section{INTRODUÇÃO}

A crescente preocupação com a preservação ambiental e o aumento da demanda por petróleo têm incentivado a busca de substitutos aos combustíveis fósseis utilizando como matérias-primas ácidos graxos e seus derivados, assim como reportado por Suarez et al. (2009). Devido a grande variedade e produtividade de óleos vegetais no Brasil, os combustíveis produzidos a partir de óleos residuais de alto teor de ácidos graxos vêm sendo uma alternativa, especialmente por não concorrer com o setor alimentício.

O craqueamento, térmico ou catalítico é uma forma de valorizar estes óleos graxos de alta acidez. Para otimizar os parâmetros de processo e maximizar os rendimentos desejados, é imprescindível o conhecimento da cinética de reação. Entretanto, há dificuldade na obtenção de dados cinéticos confiáveis no craquemaneto de matérias-primas complexas como as biomassas vide Amutio et al. (2012). Visto que, durante o processo de decomposição térmica de biomassas há reações consecutivas e paralelas, os modelos cinéticos livres têm sido utilizados no estudo de reações complexas. De acordo com Slopiecka et al. (2012), nestes métodos, a energia de ativação é obtida em função da taxa de conversão e o grau de conversão é medido por meio da perda de massa em função do aumento de temperatura em diferentes taxas de aquecimento.

Dermibas (2009) sugere a obtenção do bio-óleo a partir do craqueamento térmico de óleos vegetais, que produzirão compostos como alcenos de cadeias cíclicas e abertas, parafinas e alguns 
compostos oxigenados.

O objetivo desse trabalho foi realizar um estudo cinético do craqueamento do ácido mirístico, como molécula modelo de ácidos graxos livres, termicamente ou em presença dos catalisadores $10 \%$ $\mathrm{Mo} / \gamma-\mathrm{Al}_{2} \mathrm{O}_{3}$ e $10 \% \mathrm{Mo} / \mathrm{SiO}_{2}-\mathrm{Al}_{2} \mathrm{O}_{3}$.

\section{PARTE EXPERIMENTAL}

\subsection{Preparação e Caracterização dos catalisadores}

Os catalisadores foram obtidos por impregnação úmida dos suportes comerciais $\gamma$-alumina (Oxiteno, 99\%) e sílica-alumina, Si:Al = 9:1, (Sigma Aldrich, Grade 135) com solução aquosa de heptamolibdato de amônio tetrahidratado (HMA) [ $\left.\left(\mathrm{NH}_{4}\right)_{6} \mathrm{Mo}_{7} \mathrm{O}_{24} .4 \mathrm{H}_{2} \mathrm{O}\right](\mathrm{MERCK}, 99,9 \%)$. A massa de precursor foi calculada de forma a obter uma concentração final de $10 \%$ de $\mathrm{MoO}_{3}$ nos catalisadores calcinados. A suspensão formada pelo sal e o suporte foi agitada a $100^{\circ} \mathrm{C}$ até secagem. $\mathrm{O}$ suporte impregnado foi seco a $110^{\circ} \mathrm{C}$ e em seguida calcinado em presença de ar a $650^{\circ} \mathrm{C}$ por 7 horas com taxa de aquecimento de $5^{\circ} \mathrm{C} / \mathrm{min} . \mathrm{O} \mathrm{MoO}_{3}$ mássico, usado como referência, foi preparado via decomposição térmica do $\mathrm{HMA}$ a $550^{\circ} \mathrm{C}$ e rampa de aquecimento de $10^{\circ} \mathrm{C} / \mathrm{min}$ durante 4 horas em um forno tipo mufla.

Os catalisadores foram caracterizados por DRX em um difratômetro BRUKER D8 ADVANCE a $40 \mathrm{kV}, 40 \mathrm{~mA}$, radiação $\mathrm{CuK}_{\alpha}$ e $\lambda=1,542 \AA ̊ \AA$, na faixa de medida $2 \theta$ entre $10^{\circ}$ e $50^{\circ}$, com velocidade de passo de $0,02^{\circ}$ a cada 4,5 segundos. O teor de molibdênio foi quantificado por fluorescência de raios-X em um equipamento Shimadzu EDX-700.

O estudo da perda de massa dos catalisadores e do HMA, após secagem, foi realizado em uma termobalança Perkin Elmer STA 6000, usando uma massa de $6 \mathrm{mg}$ de material susceptível de perda de massa, num cadinho de alumina, sob fluxo de ar sintético de $20 \mathrm{~mL} / \mathrm{min}$.

\subsection{Ensaios dinâmicos}

Para o estudo da decomposição térmica e catalítica do ácido mirístico (AM) (Merck, 99,5\%) foram utilizadas quatro taxas de aquecimento $\left(\beta=3,7,10\right.$ e $\left.15{ }^{\circ} \mathrm{C} / \mathrm{min}\right)$. As condições experimentais utilizadas segundo Zanatta et al. (2012). Todos os experimentos foram conduzidos numa faixa de temperatura de $30-700^{\circ} \mathrm{C}$. No craqueamento catalítico, o AM foi misturado aos catalisadores $10 \%$ $\mathrm{Mo} / \gamma-\mathrm{Al}_{2} \mathrm{O}_{3}$ e $10 \% \mathrm{Mo} / \mathrm{SiO}_{2}-\mathrm{Al}_{2} \mathrm{O}_{3}$ numa proporção catalisador: $\mathrm{AM}$ (5:1), com excesso de catalisador para minimizar a influência do térmico. Foi realizada uma mistura mecânica do $\mathrm{AM}$ a $110^{\circ} \mathrm{C}$ no catalisador previamente seco.

O grau de conversão foi obtido a partir dos dados de perda de massa a uma taxa de aquecimento constante, conforme a Equação 1, em que $m_{t}$ representa a massa da amostra numa determinada temperatura e $m_{o}$ e $m_{f}$ são as massas da amostra inicial e final do processo, respectivamente. 


$$
\alpha=\frac{\left(m_{o}-m_{t}\right)}{\left(m_{o}-m_{f}\right)}
$$

A taxa de conversão do processo cinético pode ser descrito pela Equação 2:

$$
\frac{d \alpha}{d t}=k(T) f(\alpha)
$$

Onde $\mathrm{k}(\mathrm{T})$ é uma constante de velocidade de reação e $\mathrm{f}(\alpha)$ representa uma função que descreve a conversão da reação. Substituindo a equação de Arrhenius na Equação 2, obtém-se a Equação 3:

$$
\frac{d \alpha}{d t}=A f(\alpha) e^{-\frac{E_{0}}{R T}}
$$

Os parâmetros como energia de ativação $\left(E_{a}\right)$ e o fator pré-exponencial (A) podem ser estimados por meio da relação de Arrhenius, em que $\mathrm{R}$ e $\mathrm{T}$ são a constante dos gases e a temperatura absoluta, respectivamente.

Para condições não isotérmicas, quando a temperatura varia com o tempo e com a taxa de aquecimento constante, $\beta=\mathrm{dT} / \mathrm{dt}$, a Equação 2 é modificada:

$$
\beta \frac{d \alpha}{d t}=A f(\alpha) e^{-\frac{E_{0}}{R T}}
$$

Integrando-se a Equação 4, temos:

$$
\int_{0}^{\alpha} \frac{d \alpha}{f(\alpha)}=g(\alpha)=\frac{A}{\beta} \int_{T_{0}}^{T} e^{-\frac{E_{a}}{R T}} d T=\frac{A E_{a}}{\beta R} p(x)
$$

Onde $x=\mathrm{E} / \mathrm{RT}$. Visto que a função $p(\mathrm{x})$ não tem uma solução analítica exata, a mesma pode ser resolvida utilizando a aproximação de Doyle (1962), que é dada por: $\ln p(x)=-5,3305-1,052 x \quad(20 \leq x \leq 60)$

\subsection{Modelo de cinética livre}

O método cinético de Ozawa (1970) utiliza a aproximação de Doyle de $p(x)$ para resolver a Equação 4, para obtenção da equação linearizada, conforme Equação 6.

$$
\ln \beta_{i}=\ln \left(\frac{a_{\alpha} E_{\alpha_{i} \alpha}}{R g_{\alpha}}\right)-5,331-1,052 \frac{E_{\alpha_{i} \alpha}}{R T_{\alpha}}
$$


Assim, para uma conversão constante, o gráfico $\ln \beta_{\mathrm{i}}$ versus $\left(1000 / \mathrm{T}_{\alpha}\right)$, obtido a partir de curvas termogravimétricas registradas por meio de diferentes taxas de aquecimento $(\beta)$, fornece um modelo linearizado que permite a obtenção da energia de ativação $\left(E_{\alpha}\right)$ sem o conhecimento da ordem de reação e modelo matemático que represente a cinética do processo. O fator pré-exponencial (A) é obtido a partir do coeficiente linear dessa reta, como reportado por Flynn (1983).

\section{RESULTADOS E DISCUSSÃO}

As Figuras 1 e 2 apresentam os difratogramas do $\mathrm{MoO}_{3}$ não suportado, bem como dos suportes puros e Mo suportados em $\mathrm{SiO}_{2}-\mathrm{Al}_{2} \mathrm{O}_{3}$ e $\gamma-\mathrm{Al}_{2} \mathrm{O}_{3}$, respectivamente. De modo a melhorar a sensibilidade da análise, realizou-se uma varredura na região de $2 \theta=20-30^{\circ}$, com passos de $0,02^{\circ}$ a cada 15 segundos, nos catalisadores. Os resultados obtidos estão apresentados ao lado dos difratogramas gerais.

Na Figura 1, observa-se a presença de picos mal definidos com valores de $2 \theta=23,17^{\circ}$ e $23,44^{\circ}$, e os demais picos com valores de $25,5^{\circ}$ e 26,22 . O pico principal de $\mathrm{MoO}_{3}$, a 27,32 $2^{\circ}$ não é aparente. Os picos observados estão atribuídos à formação, no final da calcinação a $650^{\circ} \mathrm{C}$, do aluminato de molibdênio de baixa cristalinidade, assim como reportado por Debecker et al. (2011) e Kassem (2010).

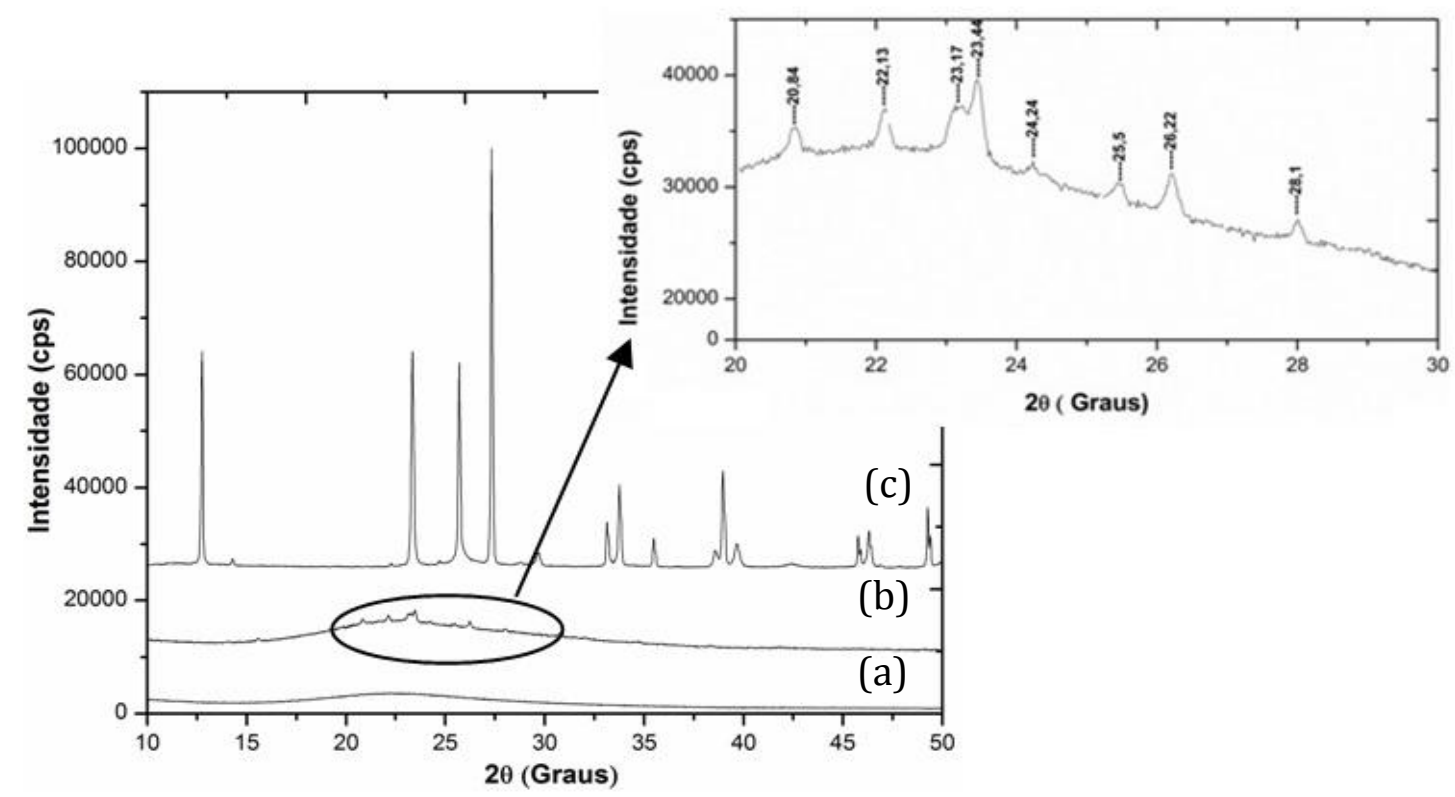

Figura 1 - Difratogramas do suporte comercial $\mathrm{SiO}_{2}-\mathrm{Al}_{2} \mathrm{O}_{3}$ (a), catalisador $10 \% \mathrm{Mo} / \mathrm{SiO}_{2}-\mathrm{Al}_{2} \mathrm{O}_{3}$ (b) e do $\mathrm{MoO}_{3}(\mathrm{c})$.

Analisando a Figura 2, na faixa entre $20-30^{\circ}$, nota-se a ausência do pico mais importante de $\mathrm{MoO}_{3}\left(27,32^{\circ}\right)$, e novamente aparecem picos que podem ser associados ao aluminato de molibdênio. 


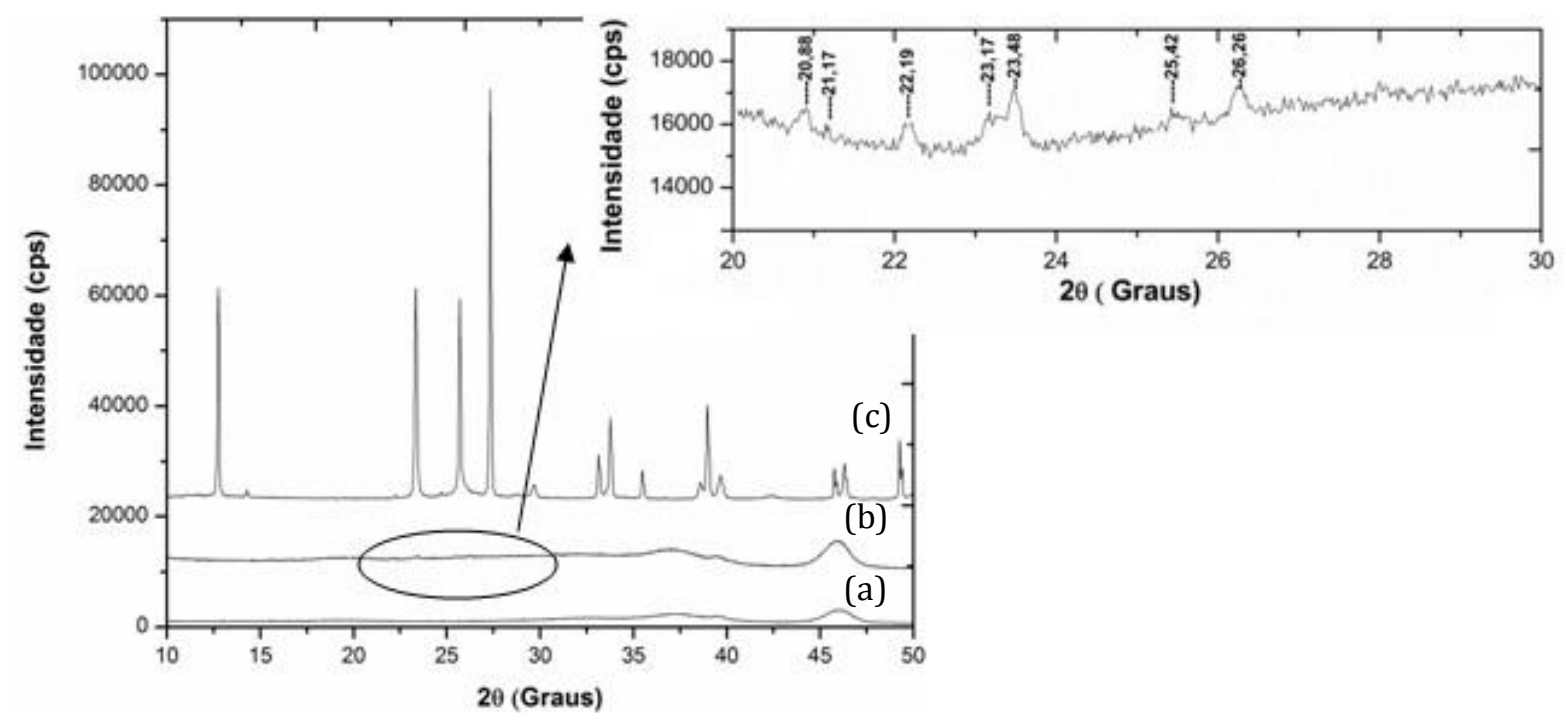

Figura 2 - Difratogramas do suporte comercial $\gamma-\mathrm{Al}_{2} \mathrm{O}_{3}$ (a), catalisador $10 \% \mathrm{Mo} / \gamma-\mathrm{Al}_{2} \mathrm{O}_{3}$ (b) e do $\mathrm{MoO}_{3}(\mathrm{c})$.

Os resultados de DRX das Figuras 1 e 2 mostram a existência da fase $\mathrm{Al}_{2}\left(\mathrm{MoO}_{4}\right)_{3}$ de simetria ortorrômbica (JCPDS 23-0764), indicada pelos picos em 20,8 ${ }^{\circ} ; 22,1^{\circ} ; 23,3^{\circ} ; 23,45^{\circ} ; 25,42^{\circ}$ e $26,2^{\circ}$ (20), corroborando com os resultados de Wanga et al. (2014) e Kassem (2006).

O resultado obtido no craqueamento térmico do ácido mirístico apresentou um único intervalo de perda de massa, podendo está associado a quebra do triglicerídeo em moléculas orgânicas menores, conforme reportado por Biswas e Sharma (2013). Os resultados de TG/DTG mostram que as perdas de massa referentes ao craqueamento catalítico do ácido mirístico misturado com $10 \%$ $\mathrm{Mo} / \gamma-\mathrm{Al}_{2} \mathrm{O}_{3}$ são apresentadas na Figura 3. As perdas globais de massa durante o aquecimento até $700^{\circ} \mathrm{C}$, para as quatro taxas de aquecimento, podem ser melhor visualizadas nas curvas DTG (Figura 3 (b)). A primeira etapa de perda, observada antes de $150^{\circ} \mathrm{C}$ é devido a perda de água adsorvida no suporte do catalisador. Ela não foi considerada para o tratamento dos dados cinéticos. Os picos DTG referenciados como 1,2,3, mostram uma tendência geral de deslocamento para maiores temperaturas, à medida que se aumenta a taxa de aquecimento $(\beta)$. Esta observação é esperada devido a dois fenômenos diferentes: i) o atraso térmico dos processos químicos quando a taxa de aquecimento é maior, e ii) a inércia da transferência de calor no sistema termogravimétrico quando a velocidade de aquecimento aumenta. $\mathrm{O}$ comportamento das curvas TG/DTG para o $\mathrm{AM}+10 \% \mathrm{Mo} / \mathrm{SiO}_{2}-\mathrm{Al}_{2} \mathrm{O}_{3}$ é comparável ao observado para o catalisador $10 \% \mathrm{Mo} / \gamma-\mathrm{Al}_{2} \mathrm{O}_{3}$ apresentado na Figura 3, por isso não foi apresentado.

O valor médio obtido para a energia de ativação no craqueamento térmico do ácido mirístico foi $98,9 \mathrm{~kJ} / \mathrm{mol}$. Para a obtenção da energia de ativação do processo catalítico foi realizado o cálculo da $E_{a}$ para cada etapa envolvida (evidenciada nas curvas de DTG), nas faixas de temperatura (1), (2) e (3) com valores de $200-250^{\circ} \mathrm{C}, 300-350^{\circ} \mathrm{C}$ e $400-450^{\circ} \mathrm{C}$, respectivamente, conforme Figura 3(b). 

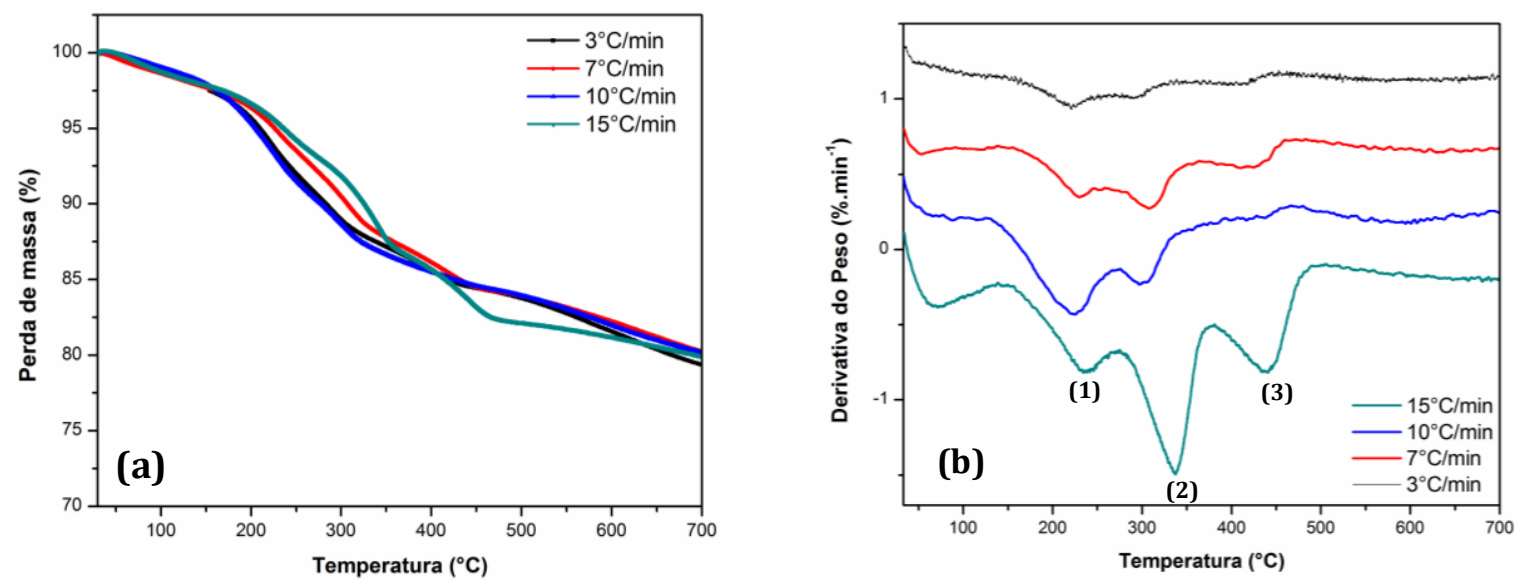

Figura 3 - Curvas TG (a) e DTG (b) para o $\mathrm{AM}+10 \% \mathrm{Mo} / \gamma-\mathrm{Al}_{2} \mathrm{O}_{3}$ para as diferentes $\beta$.

A Tabela 1 apresenta os valores obtidos para a energia de ativação (por etapas e global) na decomposição catalítica do ácido mirístico.

Tabela 1. Valores das $\mathrm{E}_{\mathrm{a}}$ obtidos para o AM misturado aos catalisadores

\begin{tabular}{|l|l|l|l|l|}
\hline \multirow{2}{*}{ Catalisadores } & \multicolumn{4}{|c|}{ Energia de ativação (kJ/mol) } \\
\cline { 2 - 5 } & $\mathbf{( 1 )}$ & $\mathbf{( 2 )}$ & $\mathbf{( 3 )}$ & Global \\
\hline $\mathrm{AM}+10 \% \mathrm{Mo} / \mathrm{Al}_{2} \mathrm{O}_{3}$ & 33,6 & 54,6 & 106,5 & 64,9 \\
\hline $\mathrm{AM}+10 \% \mathrm{Mo} / \mathrm{SiO}_{2}-\mathrm{Al}_{2} \mathrm{O}_{3}$ & 112 & 68,4 & 74,9 & 85,1 \\
\hline
\end{tabular}

A diferença entre os valores médios das energias de ativação obtidas para os diferentes catalisadores pode ser associada ao grau de interação entre a molécula do ácido em estudo e os catalisadores, seja a fase ativa, seja o próprio suporte. As medidas de infravermelho estão sendo realizadas para tentar distinguir as diferenças de adsorção da molécula de AM sobre os catalisadores.

As taxas de conversão da degradação do AM dependem da temperatura, conforme Figura 4, que mostra a conversão de degradação do $\mathrm{AM}$ puro e em presença de $10 \% \mathrm{Mo} / \gamma-\mathrm{Al}_{2} \mathrm{O}_{3}$ e $10 \%$ $\mathrm{Mo} / \mathrm{SiO}_{2}-\mathrm{Al}_{2} \mathrm{O}_{3}$. Observa-se que a degradação do $\mathrm{AM}$ é mais eficiente na presença dos catalisadores, onde suas curvas apresentam comportamento semelhante. 


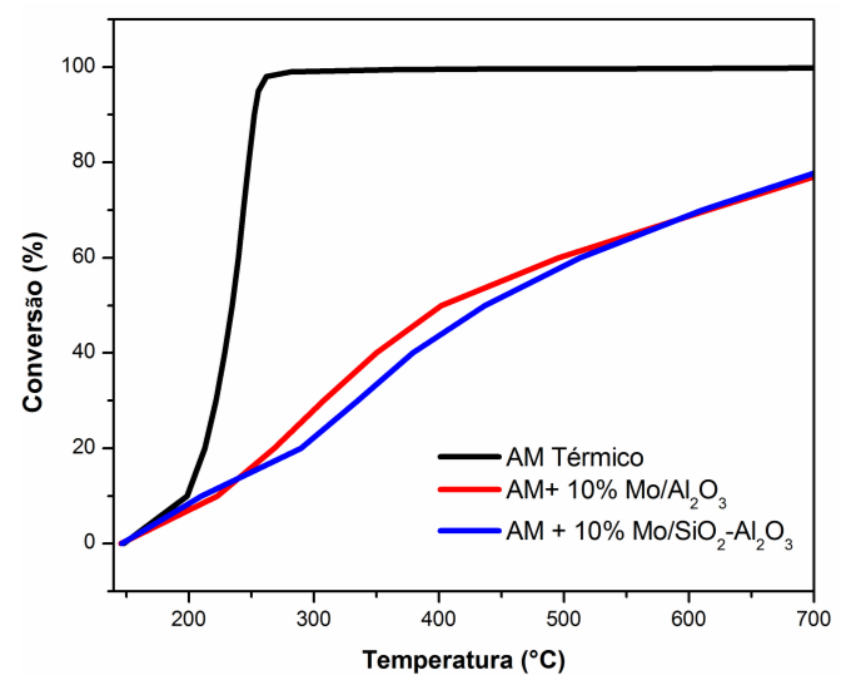

Figura 4 - Curvas de conversão do AM em função da temperatura.

\section{CONCLUSÕES}

Para os catalisadores $10 \% \mathrm{Mo} / \mathrm{SiO}_{2}-\mathrm{Al}_{2} \mathrm{O}_{3}$ e $10 \% \mathrm{Mo} / \gamma-\mathrm{Al}_{2} \mathrm{O}_{3}$, identificou-se a presença de molibdênio, porém, na forma de aluminato de molibdênio. Os resultados de TG/DTG mostram que os suportes $\gamma-\mathrm{Al}_{2} \mathrm{O}_{3}$ e $\mathrm{SiO}_{2}-\mathrm{Al}_{2} \mathrm{O}_{3}$, modificam fortemente a cinética de decomposição do sal precursor, o que sugere uma interação forte entre ambos compostos. As energias de ativação foram obtidas utilizando o modelo de FWO A degradação do AM mostrou-se mais eficiente na presença dos catalisadores, em especial $10 \% \mathrm{Mo} / \mathrm{Al}_{2} \mathrm{O}_{3}$ que apresentou menor valor para energia de ativação. $\mathrm{O}$ conhecimento de parâmetros cinéticos é importante para que modelos cinéticos úteis a nível industrial possam ser desenvolvidos.

\section{AGRADECIMENTOS}

Os autores deste trabalho agradecem à Fundação de Amparo à Ciência e Tecnologia de Pernambuco (FACEPE), ao Programa de Recursos Humanos em Petróleo e Gás Natural (PRH-28), ao Laboratório de Tecnologias e Processos Limpos (LateClim), ao Departamento de Engenharia Química (DEQ), a Universidade Federal de Pernambuco (UFPE) e ao Centro de Tecnologias Estratégicas do Nordeste (CETENE).

\section{REFERÊNCIAS}

AMUTIO, M. LOPEZ, G., AGUADO, R.,ARTETXE, M., BILBAO, J., OLAZAR, M. Kinetic study of lignocellulosic biomass oxidative pyrolysis. Fuel, v.95, n.3, p.305-311, 2012.

BISWAS, S.; SHARMA, D. Studies on cracking of Jatropha oil. J. Anal. Appl. Pyrolysis, v. 99, p. 
122-129, 2013.

DEBECKER, D. P.; SCHIMMOELLER, B.; STOYANOVA, M.; POLEUNIS, C.;BERTRAND, P.; RODEMERCK, U.; GAIGNEAUX, E. M. Flame-Made $\mathrm{MoO}_{3} / \mathrm{SiO}_{2}-\mathrm{Al}_{2} \mathrm{O}_{3}$ Metathesis Catalysts with highly Dispersed and Highly Active Molybdate Species. J. Catal., v.277, n.2, p. 154-163, 2011.

DEMIRBAS, A. Progress and recent trends in biodiesel fuels. Energy Convers. Manage., v. 50, p.14-34, 2009.

DOYLE, C.D. Estimating isothermal life from thermogravimetric data. J. Appl. Polym. Sci., v.6, p.639-642, 1962.

FLYNN, J.H. The isoconversional method for determination of energy of activation at constant heating rates. Therm. Anal. v.27, p.95-102, 1983.

KASSEM, M. Phase Relations in the $\mathrm{Al}_{2} \mathrm{O}_{3}-\mathrm{MoO}_{3}$ and $\mathrm{Al}-\mathrm{MoO}_{3}$ Systems, Investigated by X-ray Powder Diffraction, FTIR, and DTA Techniques. Inorg. Mater, v.42, n.2, p.165-170, 2006.

KASSEM, M. Reactivity in the Solid State Between Cobalt and Aluminum Molybdate. J phase equilibria diffus. v.31, n.5, p.433-438, 2010.

OZAWA, T. Kinetic Analysis of Derivative Curves in thermal Analysis, J. Thermal Anal., v.2, p.301-324, 1970.

SLOPIECKA, K.; BARTOCCI, P.; FANTOZZI,F. Thermogravimetric analysis and kinetic study of poplar wood pyrolysis. Appl. energ., v.97, p.491-497, 2012.

SUAREZ, P. A. Z.; SANTOS, A. L. F.; RODRIGUES, J. P.; ALVES, M. B. Biocombustíveis a partir de óleos e gorduras: desafios tecnológicos para viabilizá-los. Quím. Nova, v.32, n.3, p.768$775,2009$.

WANGA B.; YAO Y., JIANG M., LI Z., MA X., QIN S., SUN QI. Effect of cobalt and its adding sequence on the catalytic performance of $\mathrm{MoO}_{3} / \mathrm{Al}_{2} \mathrm{O}_{3}$ toward sulfur-resistant methanation. J. Energy Chem., v. 23, n. 1, p. 35-42, 2014.

ZANATTA, E.R.; SCHEUFELLE, F.B.;REINEHR, T.O.;SILVA, E.A.;SANTOS, J.B.O. Estudo cinético de decomposição térmica da celulose. Rev. Eletr. Cient. Inov.Tecnol.,v.1, n.5, p.29-34, 2012. 